surgery, 72(69.9\%) received PDS whilst 26(25.24\%) had Interval debulking surgery(IDS). CCR was achieved in 10(9.5\%) cases, whilst optimal and suboptimal cytoreduction was achieved in 95(90.5\%) patients. OS in patients having surgery was 42.4 months, with a 1-year survival of $83.33 \%$ and 5year survival of $27 \%$. 26(20.31\%) patients received chemotherapy only as a treatment option with an OS was 25.2 months, with a 1-year survival of $61.53 \%$ and 5 -year survival of $7.69 \%$. From the 16 patients who received best supportive care, 14/16(87.5\%) died within 6 months following diagnosis. Conclusion* The amount CCR achieved during debulking surgery was noted to be low. In order to achieve better results, it may be appropriate that AOC treatment strategy is revised, by either moving towards IDS, which we have seen in the latter 3 years, or by investing in improving the surgical expertise. Despite low levels of CCR, survival rates are comparable with other European centres.

\section{SARCOPENIA IN HIDING: CT-ASSESSED SARCOPENIA IS A PROGNOSTIC FACTOR IN OVARIAN CANCER}

${ }^{1} A$ Tranoulis*, 'A Kwong, 'A Lakhiani, ${ }^{1} R$ Gujar, ${ }^{2} D$ Georgiou, ${ }^{1} K$ Singh, ${ }^{1} \mathrm{~J}$ Balega. ${ }^{1}$ Birmingham City Hospital, UK; ${ }^{2}$ Chelsea and Westminster Hospital, UK

\subsection{6/ijgc-2021-ESGO.386}

Introduction/Background* Cancer cachexia represents a paraneoplasmatic syndrome including weight loss and sarcopenia. Sarcopenia is defined as a progressive loss of skeletal mass and function. Although poor nutritional status is adversely linked with oncological outcomes in ovarian cancer (OC), there is a paucity of data on the prevalence and prognostic value of sarcopenia in such patients. The aim of this study was to ascertain whether sarcopenia is associated with survival outcomes in OC.

Methodology MEDLINE, Scopus and Cochrane Database were searched for relevant references from inception until May 2021 in line with PRISMA guidelines. Observational studies (OSs) assessing the prevalence and prognostic impact of sarcopenia in OC were included. The methodologic index for nonrandomized studies was used to evaluate the quality of the included studies. We pooled proportions to calculate the prevalence of sarcopenia, whilst dichotomous variables were assessed using hazard ratio (HR). Confidence intervals were set at 95\%. Heterogeneity was assessed using Cochran's Q test, with an $\mathrm{I}^{2}>50 \%$ and $\mathrm{p}$-value $<0.1$ denoting significant inter-study heterogeneity. Statistical analysis was performed using the RevMan software version 5.3 and MedCalc. The level of statistical significance was set at $\mathrm{p}$-value $<0.05$.

Result(s)* Eighteen OSs were included. The studies were of moderate quality and characterised by significant clinical heterogeneity. Pooled results rendered a summary proportion of $41.91 \%$ [(95\% CI $34.97 \%$ - 49.01\%); $I^{2}=93.2 \%$ ] for the outcome of sarcopenia prevalence. Our analysis demonstrated no significant impact of sarcopenia on progression-free survival (PFS) either in univariate data $[\mathrm{HR}=1.11$, (95\% CI 0.90 1.37), $\mathrm{p}$-value $\left.=0.33 ; \mathrm{I}^{2}=45 \%\right]$ or multivariate data synthesis $\left[\mathrm{HR}=1.23, \quad(95 \%\right.$ CI $0.94 \quad-1.60), \quad \mathrm{p}$-value $\left.=0.13 ; \mathrm{I}^{2}=56 \%\right]$. Conversely, sarcopenia was significantly associated with poorer overall survival (OS) in both univariate $[\mathrm{HR}=1.27$ (95\% CI $1.05-1.54), \mathrm{p}$-value $\left.=0.02 ; \mathrm{I}^{2}=72 \%\right]$ and multivariate data synthesis $[\mathrm{HR}=1.31$ (95\% CI 1.11 - 1.55), p-value $=0.002$; $\left.\mathrm{I}^{2}=78 \%\right]$.
Conclusion* Baseline sarcopenia is seemingly an independent prognosticator in OC. Early identification and enrolment in physical and nutritional optimisation may improve oncological outcomes. Future larger prospective studies are warranted to draw firmer conclusions.

\section{UPFRONT CRS VERSUS NACT FOLLOWED BY CRS IN ADVANCED EOC IN INDIAN PATIENTS -AN ANALYTICAL RETROSPECTIVE STUDY}

A Mishra*, MD Ray, S Deo, MK Gaur, R Pramanik, S Mathur, K Luthara. All India Institute Of Medical Sciences, New Delhi, New Delhi, India

\subsection{6/ijgc-2021-ESGO.387}

Introduction/Background* Primary Cytoreductive surgery (CRS) followed by systemic chemotherapy is the standard management up to stage IIIB Epithelial ovarian cancer (EOC). The controversy starts with stage IIIC onwards ovarian cancer where differences in opinion has been noticed regarding initial approach towards these advanced cases. Incidence wise more than two third EOC patients present in advanced stages i.e., stage IIIC and beyond. As the standard treatment is primary cytoreductive surgery but it always challenges a surgeon to perform surgery in these locally advanced EOC. Therefore, the need for neoadjuvant chemotherapy (NACT) had been noticed in some selected cases. We share our outcomes as chemotherapy first or surgery first as the modality of treatment in advanced EOC.

Methodology A total of 200 patients of advanced epithelial ovarian cancer (EOC) were analyzed from 2012 to 2017 from a prospectively maintained ovarian cancer database. More than $90 \%$ patients were stage III and beyond. Overall survival was calculated in both the groups and cox multivariate analysis was performed for degree of cytoreduction and response to NACT.

Result(s)* Out of 200 included patients of advanced EOCprimary CRS was performed in 95 patients (47.5\%) and Interval CRS after 3 to 6 cycles of NACT in 105 patients $(52.5 \%)$. After median follow up of 35 months, 5 -year overall survival in upfront CRS group was $53.7 \%(\mathrm{CI}=0.405$ $0.651)$ and $\mathrm{OS}$ in NACT group was $42.2 \% \quad(\mathrm{CI}=0.318$ $0.522)$. Among upfront CRS group, optimal cytoreduction could be achieved in $66(72 \%)$ patients and in NACT group, optimal cytoreduction was achieved in $82(78 \%)$. In our tertiary care center, we offered HIPEC after CRS in both the groups where we could have achieved optimal cytoreduction.

Conclusion* Primary CRS is the standard treatment modality in advanced stages of EOC. However, in certain cohort of patient, we preferred NACT over upfront CRS. Identifying that group is challenging but feasible. Proper selection of patient is the ultimate key for reasonable outcomes.

\section{IMPACT ON SURVIVAL OF SURGICAL THERAPEUTIC STRATEGY IN THE INITIAL MANAGEMENT OF ADVANCED OVARIAN CANCER}

A Amet* ${ }^{*}$ H Costaz, JD Fumet, L Arnould, L Favier, F Beltjens, L Bengrine Lefevre, S Dabakuyo, C Coutant. 1 Rue du Professeur Marion, Dijon, France

10.1136/ijgc-2021-ESGO.388 
Introduction/Background* The management of ovarian cancer is based on a combination of surgery and chemotherapy. The aim of surgery is to achieve zero residual tumour at the end of the procedure. In advanced stage ovarian cancer, two therapeutic approaches are possible: primary debulking surgery, or primary chemotherapy followed by interval debulking surgery. The primary objective of this study was to describe overall survival (OS) in FIGO stage III and IV ovarian cancers according to the therapeutic sequence (i.e. primary surgery or interval surgery).

Methodology We performed a retrospective, observational study using data from the gynecological cancer registry of the Cote d'Or, for patients diagnosed with FIGO stage III or IV ovarian cancer between 1998 and 2015. We recorded FIGO stage, histological type, treatment and completeness of cytoreduction.

Result(s)* In total, 460 patients were included. OS at 5 years was $47 \%$ in patients with primary surgery, versus $38 \%$ in patients with interval surgery $(p=0.06)$. Five-year OS was $45 \%$ in patients with complete cytoreduction, versus $30 \%$ in those with incomplete cytoreduction $(p<0.001)$. The rate of complete cytoreduction was $43 \%$ in patients with primary surgery, versus $55 \%$ in those with interval surgery.

Conclusion* OS appears to be slightly better in patients receiving primary surgery, and when cytoreduction is complete. Every effort should be made during surgery to achieve complete cytoreduction, by an experienced team. Primary surgery should be preferred in these patients.

\section{NOVEL STONY BROOK TAXANES ARE EFFICACIOUS IN PACLITAXEL-RESISTANT OVARIAN CANCER MODELS BOTH IN VITRO AND IN VIVO}

1,2,3 P Holÿ ${ }^{*},{ }^{1,3} \mathrm{~K}$ Seborova, ${ }^{1,2,3} \mathrm{~A}$ Spalenkova, ${ }^{1,3} \mathrm{~K}$ Koucka, $1,3 \mathrm{M}$ Ehrlichova, ${ }^{4} \mathrm{C}$ Wang, ${ }^{4}$ I Ojima, ${ }^{5} \mathrm{P}$ Daniel, ${ }^{5} \mathrm{~K}$ Balusikova, ${ }^{5} \mathrm{M}$ Jelinek, ${ }^{5} \mathrm{~J}$ Kovar, ${ }^{1,3} \mathrm{P}$ Soucek, ${ }^{1,3} \mathrm{R}$ Vaclavikova. ${ }^{1}$ Biomedical Center, Charles University Faculty of Medicine in Pilsen, Laboratory of Pharmacogenomics, Pilsen, Czech Republic; ${ }^{2}$ Third Faculty of Medicine, Charles University, Prague, Czech Republic; ${ }^{3}$ National Institute of Public Health, Prague, Toxicogenomics Unit, Prague, Czech Republic; ${ }^{4}$ Institute of Chemical Biology and Drug Discovery - Stony Brook University - State University of New York, Stony Brook, NY, USA; ${ }^{5}$ Third Faculty of Medicine, Charles University, Division of Cell and Molecular Biology, Prague, Czech Republic

\subsection{6/ijgc-2021-ESG0.389}

Introduction/Background* Resistance of cancer cells to taxanes is a serious problem preventing successful therapy. Efforts are ongoing to synthesize novel taxanes efficacious against the resistant phenotype. Stony Brook taxanes (SB-Ts) have proven to have potential, but require further preclinical testing and more detailed study of their mechanism of action. Here, we aimed to evaluate the efficacy of several promising SB-Ts in resistant ovarian cancer models in vitro, and in vivo. We also studied the role of 3 candidate genes ABCC3, CPS1, and TRIP6 in SB-Ts cell death-inducing molecular mechanisms.

Methodology The NCI/ADR-RES ovarian cancer cell line was incubated with either paclitaxel or one of the second generation (SB-T-1214 and SB-T-1216) or third generation (SB-T121402, SB-T-121605 and SB-T-121606) taxanes. Cell survival was measured as IC50 after 72 hours. Cell cycle analysis was performed using flow cytometry. Uptake of SB-Ts into cells was measured by HPLC. Female athymic mice Nude Crl: NU (NCr)-Foxn1nu $(\mathrm{N}=50)$ were used as the model organism for ovarian cancer by subcutaneous application of NCI/ADR-RES cells. In vivo efficacy of taxanes was measured after intraperitoneal application twice per week. Gene expression in tumour tissue was measured by RT-qPCR.

Result(s)* Compared to paclitaxel, NCI/ADR-RES cells showed $30 \mathrm{x}$ lower resistance to SB-T-1214, SB-T-1216, and SB-T121402 and $50 \mathrm{x}$ lower to the 3rd generation taxanes SB-T121605 and SB-T-121606. Cell cycle analysis showed the "1216" family to be more cytostatic than cytotoxic compared to "1214". Uptake of SB-T-1216 and its derivatives into cells was $6-15.5 \mathrm{x}$ higher than for paclitaxel. Treatment of mice (groups of 5) with regimen combining paclitaxel and SB-T121605/-06 significantly slowed tumour growth and even reduced tumour volume after several applications. Doses of SB-Ts higher than $3 \mathrm{mg} / \mathrm{kg}$ caused severe toxicity. Both SB-T121605 and SB-T-121606, but not paclitaxel, led to significant decrease in CPS1 and ABCC3 expression in vitro and in case of CPS1 also in vivo.

Conclusion* Third generation taxanes SB-T-121605 and SB-T121606 are highly effective in a paclitaxel-resistant ovarian carcinoma model both in vitro and in vivo and warrant further investigation. SB-T treatment led to deregulation of CPS1 and $\mathrm{ABCC} 3$ expression that seems to play a role in their efficacy.

\section{LONG TERM PROGNOSIS OF PREMENOPAUSAL WOMEN WITH OVARIAN CANCER}

V Corraliza-Galan*, C Martin-Gromaz, I Pelayo, D Rubio-Marin, E Cabezas-Lopez, C Del Valle-Rubido, MJ Pablos-Antona, L Abarca-Martinez, E Moratalla-Bartolome, C SanchezMartinez, J Lazaro de la Fuente. Ramon y Cajal Hospital , Obstetrics and Gynecology, Madrid, Spain

\subsection{6/ijgc-2021-ESGO.390}

Introduction/Background* Ovarian cancer (OC) is the most lethal gynaecological malignancy worldwide. In general, patients face a poor prognosis due to the fact that they often have an advanced stage of disease at diagnosis. The peak incidence is seen at 65 to 70 years and only a small group of women is diagnosed under 40 years of age. Younger women have better overall survival compared to older women but prognostic factors and evolution are not well stablished.

Methodology Retrospective analysis of women under 45 years old diagnosed of epithelial and non-epithelial ovarian cancer during the last 10 years.

Result(s)* 25 women under 45 years with OC were reviewed. Mean age at diagnosis was 36.27 years (SD 5.77; min:21, max: 43). Most of the tumors (52\% N:13) were epithelial serous OC (Clear cells: 20.0\% N:5; Endometrioid: 12.0 $\mathrm{N}: 3$; Mucinous: $8.0 \% \mathrm{~N}: 2$; Endodermal sinus: $4.0 \% \mathrm{~N}: 1$; Granulosa cell: $4.0 \% \mathrm{~N}: 1$ ). Most of the patients were diagnosed in advanced tumoral stages (III-IV: 68.0\%, N:17). Appropriate surgery and chemotherapy was applied individually in each case. After a long period of follow up (6-108 months) $50 \%$ of women were death (medium follow up for alive women: 66.44 months (SD: 26.93; min: 24; max: 108 months; medium follow up for death women: 23.60 months; SD: 14.45 ; min:6; max: 41 months). Mean time of relapse was 14.33 moths (SD: 12.30; min: 4; max: 45 months), mostly in abdominal location $(92.85 \% \mathrm{~N}: 13)$, that were treated with quemotherapy $(85.71 \% \quad \mathrm{~N}: 12)$ and surgery $(28.75 \% \mathrm{~N}: 4)$. After relapse only $20.0 \%$ were disease free $(\mathrm{N}: 3)$ while 2 patients died and $60.0 \%$ (N: 15) were alive with disease. 